\title{
A multivalued version of Krasnoselskii's theorem in generalized Banach spaces
}

\author{
Ioan-Radu PETRE
}

\begin{abstract}
The purpose of this paper is to extend Krasnoselskii's fixed point theorem to the case of generalized Banach spaces for multivalued operators. As application, we will give an existence result for a system of Fredholm-Volterra type differential inclusions.
\end{abstract}

\section{Introduction, notations and auxiliary results}

It is well known that A.I. Perov (see [17]) extended the classical Banach contraction principle in the setting of spaces endowed with vector-valued metrics (see also A.I. Perov, A.V. Kibenko [18]).

Also, is known that Krasnoselskii's theorem combine a metrical fixed point result (a contraction principle) and a topological one (a Schauder-type theorem). Moreover, the multivalued forms of Krasnoselskii's theorem deals with the notion of selection. Auxiliary results are needed to ensure that a multivalued operator admits a continuous selection. There is also a vast literature concerning on this aspects in nonlinear analysis, see, for example, [3], [5], [9], [12], [16], [19], [20], [21], [22], [29], [36], [39], [40].

Key Words: Compact operator, fixed point, generalized contraction, generalized Banach space, generalized metric space, integral inclusions system, iterative method, Krasnoselskii's theorem, $A$-contraction, matrix convergent to zero, multivalued operator, Perov's theorem, relatively compact operator, sum of two operators, vector-valued metric, vector-valued norm, Fredholm-Volterra type inclusions system.

2010 Mathematics Subject Classification: Primary 47H10, 54H25.

Received: August, 2012.

Revised: November, 2012.

Accepted: February, 2013. 
Thus, it is obvious that Krasnoselskii's theorem represents an important abstract tool in the study of differential and integral inclusions systems. Therefore, the aim of this paper is to present a multivalued version of Krasnoselskii's theorem in vector Banach spaces and a nice existence result for a FredholmVolterra type integral inclusions system.

Now, we recall some basic results (see [2], [8] and [28]), which are needed for the main results of this paper. Notice that in [28] and [8], are pointed out some advantages of a vector-valued norm with respect to the usual scalar norms.

Definition 1.1. ([17]) Let $X$ be a nonempty set and consider the space $\mathbb{R}_{+}^{m}$ endowed with the usual component-wise partial order. The mapping $d: X \times X \rightarrow \mathbb{R}_{+}^{m}$ which satisfies all the usual axioms of the metric is called a generalized metric in the Perov's sense and $(X, d)$ is called a generalized metric space.

Let $(X, d)$ be a generalized metric space in Perov's sense. Thus, if $v, r \in$ $\mathbb{R}^{m}, v:=\left(v_{1}, v_{2}, \ldots, v_{m}\right)$ and $r:=\left(r_{1}, r_{2}, \ldots, r_{m}\right)$, then by $v \leq r$ we mean $v_{i} \leq r_{i}$, for each $i \in\{1,2, \ldots, m\}$ and by $v<r$ we mean $v_{i}<r_{i}$, for each $i \in\{1,2, \ldots, m\}$. Also, $|v|:=\left(\left|v_{1}\right|,\left|v_{2}\right|, \ldots,\left|v_{m}\right|\right)$.

If $u, v \in \mathbb{R}^{m}$, with $u:=\left(u_{1}, u_{2}, \ldots, u_{m}\right)$ and $v:=\left(v_{1}, v_{2}, \ldots, v_{m}\right)$, then $\max (u, v):=\left(\max \left(u_{1}, v_{1}\right), \ldots, \max \left(u_{m}, v_{m}\right)\right)$. If $c \in \mathbb{R}$, then $v \leq c$ means $v_{i} \leq c$, for each $i \in\{1,2, \ldots, m\}$. Notice that, through this paper, we will make an identification between row and column vectors in $\mathbb{R}^{m}$. Let $(X, d)$ be a generalized metric space in Perov's sense. For $r:=\left(r_{1}, \cdots, r_{m}\right) \in \mathbb{R}^{m}$ with $r_{i}>0$ for each $i \in\{1,2, \cdots, m\}$, we will denote by

$$
B\left(x_{0}, r\right):=\left\{x \in X: d\left(x_{0}, x\right)<r\right\}
$$

the open ball centered in $x_{0}$ with radius $r$ and by

$$
\bar{B}\left(x_{0}, r\right):=\left\{x \in X: d\left(x_{0}, x\right) \leq r\right\}
$$

the closed ball centered in $x_{0}$ with radius $r$.

We mention that for generalized metric spaces in Perov's sense, the notions of convergent sequence, Cauchy sequence, completeness, open subset and closed subset are similar to those for usual metric spaces.

Definition 1.2. A square matrix of real numbers is said to be convergent to zero if and only if its spectral radius $\rho(A)$ is strictly less than 1 . In other words, this means that all the eigenvalues of $A$ are in the open unit disc, i.e., $|\lambda|<1$, for every $\lambda \in \mathbb{C}$ with $\operatorname{det}(A-\lambda I)=0$, where $I$ denotes the unit matrix of $\mathcal{M}_{m, m}(\mathbb{R})$ (see [35]). 
For a matrix $A:=\left(a_{i j}\right)_{i, j \in\{1, \cdots, m\}} \in \mathcal{M}_{m, m}(\mathbb{R})$ we denote by

$$
|A|:=\left(\left|a_{i j}\right|\right)_{i, j \in\{1, \cdots, m\}} \in \mathcal{M}_{m, m}\left(\mathbb{R}_{+}\right) .
$$

In this context, we say that a non-singular matrix $A$ has the absolute value property if $A^{-1}|A| \leq I$. Some examples of matrices convergent to zero $A \in$ $\mathcal{M}_{m, m}(\mathbb{R})$, which also satisfies the property $(I-A)^{-1}|I-A| \leq I$ are:

1) $A=\left(\begin{array}{cc}a & 0 \\ 0 & b\end{array}\right)$, where $a, b \in \mathbb{R}_{+}$and $\max (a, b)<1$;

2) $A=\left(\begin{array}{ll}a & -c \\ 0 & b\end{array}\right)$, where $a, b, c \in \mathbb{R}_{+}$and $a+b<1, c<1$;

3) $A=\left(\begin{array}{cc}a & -a \\ b & -b\end{array}\right)$, where $a>1, b>0$ and $|a-b|<1$.

In particular, if $E$ is a linear space, then $\|\cdot\|: E \rightarrow \mathbb{R}_{+}^{m}$ is a vector-valued norm if (in a similar way to the vector-valued metric) it satisfies the classical axioms of a norm. In this case, the pair $(E,\|\cdot\|)$ is called a generalized normed space. If the generalized metric generated by the norm $\|\cdot\|$ (i.e., $d(x, y):=\|x-y\|)$ is complete then the space $(E,\|\cdot\|)$ is called a generalized Banach space.

In the context of a generalized metric space $(X, d)$, we will use the following notations and definitions.

$P(X)$ - the set of all nonempty subsets of $X$;

$\mathcal{P}(X)=P(X) \cup\{\emptyset\}$

$P_{b}(X)$ - the set of all nonempty bounded subsets of $X$;

$P_{b, c l}(X)$ - the set of all nonempty bounded and closed subsets of $X$;

If $(X,\|\cdot\|)$ is a generalized normed space, then:

$P_{b, c l, c v}(X)$ - the set of all nonempty bounded, closed and convex subsets of $X$;

$P_{c p, c v}(X)$ - the set of all nonempty compact and convex subsets of $X$.

Let $(X, d)$ be a metric space. Then we introduce the following functionals.

$D_{d}: P(X) \times P(X) \rightarrow \mathbb{R}_{+}, D_{d}(A, B)=\inf \{d(a, b): a \in A, b \in B\}$ - the gap functional;

$\rho_{d}: P(X) \times P(X) \rightarrow \mathbb{R}_{+} \cup\{+\infty\}, \rho_{d}(A, B)=\sup \{D(a, B): a \in A\}$ - the excess functional;

$H_{d}: P(X) \times P(X) \rightarrow \mathbb{R}_{+} \cup\{+\infty\}, H_{d}(A, B)=\max \{\rho(A, B), \rho(B, A)\}-$ the Pompeiu-Hausdorff functional. 
If $(X, d)$ is a generalized metric space with $d(x, y):=\left(\begin{array}{c}d_{1}(x, y) \\ \cdots \\ d_{m}(x, y)\end{array}\right)$, then we denote by

$$
D(A, B):=\left(\begin{array}{c}
D_{d_{1}}(A, B) \\
\cdots \\
D_{d_{m}}(A, B)
\end{array}\right) \text { the vector gap functional on } P(X),
$$

by

$$
\rho(A, B):=\left(\begin{array}{c}
\rho_{d_{1}}(A, B) \\
\ldots \\
\rho_{d_{m}}(A, B)
\end{array}\right) \text { the vector excess functional, }
$$

and by

$$
H(A, B):=\left(\begin{array}{c}
H_{d_{1}}(A, B) \\
\cdots \\
H_{d_{m}}(A, B)
\end{array}\right) \text { the vector Pompeiu-Hausdorff functional. }
$$

For a multivalued operator $F: X \rightarrow P(X)$, we denote by $\operatorname{Fix}(F)$ the fixed point set of $F$, i.e., $\operatorname{Fix}(F):=\{x \in X \mid x \in F(x)\}$. The symbol $\operatorname{Graph}(F):=\{(x, y) \in X \times X: y \in F(x)\}$ denotes the graph of $F$.

For some topological aspects in generalized metric spaces see, for example, [32], [9], [38], [39]. We recall now the following Schauder type theorem.

Theorem 1.3. ([9]) Let $(X,\|\cdot\|)$ be a generalized Banach space, let $Y \in$ $P_{c v}(X)$ and $g: Y \rightarrow Y$ be a continuous operator with relatively compact range. Then $g$ has at least one fixed point in $Y$.

If $(X, d)$ is a generalized metric space, then $F: X \rightarrow P(X)$ is said to be a multivalued weak Picard operator if, for each $x \in X$ and $y \in F(x)$, there exists a sequence $\left(x_{n}\right)_{n \in \mathbb{N}}$ such that:

i) $x_{0}=x, x_{1}=y$;

ii) $x_{n+1} \in F\left(x_{n}\right)$;

iii) the sequence $\left(x_{n}\right)_{n \in \mathbb{N}}$ is convergent to a fixed point of $F$.

A sequence $\left(x_{n}\right)_{n \in \mathbb{N}}$ satisfying (i) and (ii) is said to be a sequence of successive approximations for $F$ starting from $\left(x_{0}, x_{1}\right) \in \operatorname{Graph}(F)$. 
Definition 1.4. ([2]) Let $(X, d)$ be a generalized metric space, $Y \subset X$ and $F: Y \rightarrow P(X)$ be a multivalued operator. Then, $F$ is called a multivalued $A$-contraction if and only if, $A \in \mathcal{M}_{m, m}\left(\mathbb{R}_{+}\right)$is a matrix convergent to zero and for any $x, y \in Y$ and for each $u \in F(x)$, there exists $v \in T(y)$ such that

$$
d(u, v) \leq A d(x, y) .
$$

Notice now that using the generalized Pompeiu-Hausdorff functional on $P_{b, c l}(X)$, the concept of multivalued contraction mapping introduced by S.B. Nadler Jr. can be extended to generalized metric spaces in the sense of Perov.

Definition 1.5. ([2]) Let $(X, d)$ be a generalized metric space, $Y \subseteq X$ and let $F: Y \rightarrow P_{b, c l}(X)$ be a multivalued operator. Then, $F$ is called a multivalued $A$-contraction in the sense of Nadler if and only if, $A \in \mathcal{M}_{m, m}\left(\mathbb{R}_{+}\right)$is a matrix convergent to zero and

$$
H(F(x), F(y)) \leq A d(x, y), \text { for any } x, y \in Y .
$$

If $X, Y$ are two generalized metric spaces, then a multivalued operator $F: X \rightarrow P(Y)$ is said to be:

a) lower semi-continuous (briefly l.s.c.) in $x_{0} \in X$ if and only if, for any open set $U \subset X$ such that $F\left(x_{0}\right) \cap U \neq \emptyset$, there exists a neighborhood $V$ for $x_{0}$ such that for any $x \in V$, we have that $F(x) \cap U \neq \emptyset$.

b) Hausdorff lower semi-continuous (briefly $H$-l.s.c.) in $x_{0} \in X$ if and only if, for any $\varepsilon=\left(\varepsilon_{1}, \cdots, \varepsilon_{m}\right) \in \mathbb{R}_{+}^{m}$ with $\varepsilon_{i}>0$ for each $i \in\{1, \cdots, m\}$, there exists $\eta=\left(\eta_{1}, \cdots, \eta_{m}\right) \in \mathbb{R}_{+}^{m}$ with $\eta_{i}>0$ for each $i \in\{1, \cdots, m\}$, such that for any $x \in B\left(x_{0}, \eta\right)$, we have $F\left(x_{0}\right) \subset V(F(x) ; \varepsilon)$, where

$$
V(F(x) ; \varepsilon)=\{x \in X: D(x, F(x)) \leq \varepsilon\} .
$$

Now, we recall several auxiliary results proved in paper [19].

Theorem 1.6. ([19]) Let $(X, d)$ be a complete generalized metric space and $F: X \rightarrow P_{c l}(X)$ be a multivalued A-contraction in Nadler's sense. Then, for each $x \in X$ and $y \in F(x)$, there exists a sequence $\left(x_{n}\right)_{n \in \mathbb{N}}$ of successive approximations for $F$ starting from $(x, y) \in \operatorname{Graph}(F)$, which converge to a fixed point $x^{*} \in X$ of $F$ and we have the following estimations:

(a) $d\left(x_{n}, x^{*}\right) \leq A^{n}(I-A)^{-1} d\left(x_{0}, x_{1}\right)$, for any $n \in \mathbb{N}^{*}$.

(b) $d\left(x_{0}, x^{*}\right) \leq(I-A)^{-1} d\left(x_{0}, x_{1}\right)$.

Another useful result for our aim is the following data dependence lemma. 
Lemma 1.7. ([19]) Let $(X, d)$ be a complete generalized metric space and $F_{1}, F_{2}: X \rightarrow P_{b, c l}(X)$ be two multivalued A-contractions in Nadler' sense. Then:

$$
\rho\left(\operatorname{Fix}\left(F_{1}\right), \operatorname{Fix}\left(F_{2}\right)\right) \leq(I-A)^{-1}\left(\begin{array}{c}
\sup _{x \in X} \rho_{d_{1}}\left(F_{1}(x), F_{2}(x)\right) \\
\ldots \\
\sup _{x \in X} \rho_{d_{m}}\left(F_{1}(x), F_{2}(x)\right)
\end{array}\right) .
$$

Also, we need an extended result of a Rybinski-type selection theorem.

Theorem 1.8. ([19]) Let $(X, d)$ be a generalized metric space and $Y$ be a closed subset of a generalized Banach space $(Z,\|\cdot\|)$. Assume that the multivalued operator $F: X \times Y \rightarrow P_{c l, c v}(Y)$ satisfies the following conditions:

i) A is a matrix convergent to zero and

$$
H\left(F\left(x, y_{1}\right), F\left(x, y_{2}\right)\right) \leq A\left\|y_{1}-y_{2}\right\|, \text { for each }\left(x, y_{1}\right),\left(x, y_{2}\right) \in X \times Y ;
$$

ii) for every $y \in Y, F(\cdot, y)$ is $H$-l.s.c. on $X$.

Then there exists a continuous mapping $f: X \times Y \rightarrow Y$ such that:

$$
f(x, y) \in F(x, f(x, y)) \text {, for each }(x, y) \in X \times Y .
$$

Lemma 1.9. ([32]) Let $(X,\|\cdot\|)$ be a generalized Banach space. Then:

$$
H(Y+Z, Y+W) \leq H(Z, W) \text {, for each } Y, Z, W \in P_{b}(X) .
$$

We recall that a measurable multivalued operator $F:[a, b] \rightarrow P_{c p}\left(\mathbb{R}^{n}\right)$ is said to be integrably bounded if and only if, there exists a Lebesgue integrable function $m:[a, b] \rightarrow \mathbb{R}^{n}$ such that for each $v \in F(t)$, we have $\|v\| \leq m(t)$, a.e. on $[a, b]$. For a measurable and integrably bounded multivalued operator $F$, the set $S_{F}^{1}$ of all Lebesgue integrable selections for $F$ is closed and nonempty (see $[6])$.

\section{Main results}

In this section, we prove a Krasnoselskii type fixed point theorem in generalized Banach spaces for a sum of two multivalued operators, where one of the operators satisfies a multivalued $A$-contraction condition in Nadler's sense and the other operator satisfies a compactness condition. Also, an application to a Fredholm-Volterra type differential inclusions system is given here. Notice that both results deals with the absolute value property of matrixes that converges to zero. 
Theorem 2.1. Let $(X,\|\cdot\|)$ be a generalized Banach space and $Y \in P_{b, c l, c v}(X)$. Assume that the operators $F: Y \rightarrow P_{b, c l, c v}(X)$, $G: Y \rightarrow P_{c p, c v}(X)$ satisfies the properties:

i) $F\left(y_{1}\right)+G\left(y_{2}\right) \subset Y$, for each $y_{1}, y_{2} \in Y$;

ii) F is a multivalued A-contraction mapping in Nadler's sense;

iii) $G$ is l.s.c and $G(Y)$ is relatively compact;

iv) the matrix I - A has the absolute value property.

Then $F+G$ has a fixed point in $Y$.

Proof. We show that for any $x \in Y$, the operator $T_{x}: Y \rightarrow P_{c p, c v}(Y)$, $T_{x}(y)=F(y)+G(x)$ is a multivalued $A$-contraction. We have that

$$
\begin{aligned}
H\left(T_{x}\left(y_{1}\right), T_{x}\left(y_{2}\right)\right) & =H\left(F\left(y_{1}\right)+G(x), F\left(y_{2}\right)+G(x)\right) \\
& \leq H\left(F\left(y_{1}\right), F\left(y_{2}\right)\right) \leq A\left\|y_{1}-y_{2}\right\|, \text { for any } y_{1}, y_{2} \in Y .
\end{aligned}
$$

Thus, $T_{x}$ is a multivalued $A$-contraction. By Theorem 1.6, it follows that for any $x \in Y$ the fixed point set for the multivalued operator $T_{x}$, Fix $\left(T_{x}\right)=$ $\{y \in F(y)+G(x)\}$ is nonempty and closed.

Since, the multivalued operator

$$
U: Y \times Y \rightarrow P_{c p, c v}(Y), U(x, y)=F(y)+G(x)
$$

satisfies the hypothesis of Theorem 1.8, there exists a continuous mapping $u: Y \times Y \rightarrow Y$ such that $u(x, y) \in F(u(x, y))+G(x)$, for each $(x, y) \in Y \times Y$.

We define $C: Y \rightarrow P_{c l}(Y), C(x)=\operatorname{Fix}\left(T_{x}\right)$ and we consider the singlevalued operator $c: Y \rightarrow Y, c(x)=u(x, x)$, for each $x \in Y$.

Now, we prove that $c(Y)$ is relatively compact. For this purpose it is sufficient to show that $C(Y)$ is relatively compact. Since $G(Y)$ is relatively compact, we have that $G(Y)$ is also totally bounded (see [38], pp. 500). Thus, for any $\varepsilon \in \mathbb{R}_{+}^{m}$ (with $\varepsilon_{i}>0$ for each $i \in\{1, \ldots, m\}$ ), there exists $Z=\left\{x_{1}, \ldots, x_{n}\right\} \subset Y$ such that $G(Y) \subset\left\{z_{1}, \ldots, z_{n}\right\}+\bar{B}(0,|I-A| \varepsilon) \subset$ $\left\{G\left(x_{1}\right), \ldots, G\left(x_{n}\right)\right\}+\bar{B}(0,|I-A| \varepsilon)$, where $z_{i} \in G\left(x_{i}\right)$ for any $i \in\{1,2, \ldots, n\}$.

It follows that, for any $x \in Y, G(x) \subset \bigcup_{i=1}^{n} G\left(x_{i}\right)+\bar{B}(0,|I-A| \varepsilon)$ and thus, 
there exists an element $x_{k} \in Z$ such that $\rho\left(G(x), G\left(x_{k}\right)\right) \leq|I-A| \varepsilon$. Then

$$
\begin{aligned}
\rho\left[C(x), C\left(x_{k}\right)\right] & =\rho\left[\operatorname{Fix}\left(T_{x}\right), \operatorname{Fix}\left(T_{x_{k}}\right)\right] \\
& \leq(I-A)^{-1}\left(\begin{array}{c}
\sup _{y \in Y} \rho_{d_{1}}\left[T_{x}(y), T_{x_{k}}(y)\right] \\
\cdots \\
\sup _{y \in Y} \rho_{d_{m}}\left[T_{x}(y), T_{x_{k}}(y)\right]
\end{array}\right) \\
& =(I-A)^{-1}\left(\begin{array}{l}
\sup _{y \in Y} \rho_{d_{1}}\left[F(y)+G(x), F(y)+G\left(x_{k}\right)\right] \\
\sup _{y \in Y} \rho_{d_{m}}\left[F(y)+G(x), F(y)+G\left(x_{k}\right)\right]
\end{array}\right) \\
& \leq(I-A)^{-1}\left(\begin{array}{l}
\sup _{y \in Y} \rho_{d_{1}}\left[G(x), G\left(x_{k}\right)\right] \\
\sup _{y \in Y} \rho_{d_{m}}\left[G(x), G\left(x_{k}\right)\right]
\end{array}\right) \\
& \leq(I-A)^{-1}|I-A| \varepsilon=\varepsilon .
\end{aligned}
$$

It follows that for any $v \in C(x)$, there exists $w_{k} \in C\left(x_{k}\right)$ such that $\left\|v-w_{k}\right\| \leq$ $\varepsilon$. Thus, for any $x \in Y, C(x) \subset \bigcup_{k=1}^{n} \bar{B}\left(w_{k}, \varepsilon\right)$. So, $C(Y)$ is relatively compact. Moreover, the operator $c: Y \rightarrow Y$ satisfies the assumptions of Theorem 1.3. Let $x^{*} \in Y$ be a fixed point for $c$. Hence, we have that $x^{*}=c\left(x^{*}\right) \in$ $F\left(c\left(x^{*}\right)\right)+G\left(x^{*}\right)=F\left(x^{*}\right)+G\left(x^{*}\right)$.

Now, using Theorem 2.1 we can obtain a nice existence result for a system of Fredholm-Volterra type integral inclusions.

Theorem 2.2. Let $I=[0, a]$ (with $a>0$ ) be an interval of the real axis and let us consider the following inclusions system in $C\left(I, \mathbb{R}^{n}\right) \times C\left(I, \mathbb{R}^{p}\right)$ :

$$
\left\{\begin{array}{l}
x_{1}(t) \in \lambda_{11} \int_{0}^{t} K_{1}\left(t, s, x_{1}(s), x_{2}(s)\right) d s+\lambda_{12} \int_{0}^{a} L_{1}\left(t, s, x_{1}(s), x_{2}(s)\right) d s \\
x_{2}(t) \in \lambda_{21} \int_{0}^{t} K_{2}\left(t, s, x_{1}(s), x_{2}(s)\right) d s+\lambda_{22} \int_{0}^{a} L_{2}\left(t, s, x_{1}(s), x_{2}(s)\right) d s
\end{array}\right.
$$

for $t \in I$, where $\lambda_{i j} \in \mathbb{R}, i, j \in\{1,2\}$. We assume that:

i) $K_{1}: I^{2} \times \mathbb{R}^{n} \times \mathbb{R}^{p} \rightarrow P_{c l, c v}\left(\mathbb{R}^{n}\right), K_{2}: I^{2} \times \mathbb{R}^{n} \times \mathbb{R}^{p} \rightarrow P_{c l, c v}\left(\mathbb{R}^{p}\right)$ are two l.s.c., measurable and integrable bounded multivalued operators;

ii) $L_{1}: I^{2} \times \mathbb{R}^{n} \times \mathbb{R}^{p} \rightarrow P_{c p, c v}\left(\mathbb{R}^{n}\right), L_{2}: I^{2} \times \mathbb{R}^{n} \times \mathbb{R}^{p} \rightarrow P_{c p, c v}\left(\mathbb{R}^{p}\right)$ are two l.s.c., measurable and integrable bounded (by two integrable functions $\left.m_{L_{1}}, m_{L_{2}}\right)$ multivalued operators; 
iii) there exists the matrix $A=\left(\begin{array}{ll}a_{11} & a_{12} \\ a_{21} & a_{22}\end{array}\right) \in M_{2,2}\left(\mathbb{R}_{+}\right)$such that for each $\left(t, s, u_{1}, u_{2}\right),\left(t, s, v_{1}, v_{2}\right) \in I^{2} \times \mathbb{R}^{n} \times \mathbb{R}^{p}$ and for $i \in\{1,2\}$, we have:

$$
H\left(K_{i}\left(t, s, u_{1}, u_{2}\right), K_{i}\left(t, s, v_{1}, v_{2}\right)\right) \leq a_{i 1}\left|u_{1}-v_{1}\right|+a_{i 2}\left|u_{2}-v_{2}\right| ;
$$

iv) $\left(\begin{array}{c}\left|\lambda_{11}\right| \\ \left|\lambda_{21}\right|\end{array}\right) \leq\left(\begin{array}{c}\frac{R_{1}}{2 a\left(a_{11} R_{1}+a_{12} R_{2}\right)} \\ \frac{R_{2}}{2 a\left(a_{21} R_{1}+a_{22} R_{2}\right)}\end{array}\right)$ and $\left(\begin{array}{c}\left|\lambda_{12}\right| \\ \left|\lambda_{22}\right|\end{array}\right) \leq\left(\begin{array}{c}\frac{R_{1}}{2 M_{L_{1}} a} \\ \frac{R_{2}}{2 M_{L_{2}} a}\end{array}\right)$, where

$$
M_{L_{1}}=\max _{t, s \in[0, a]}\left|m_{L_{1}}\right|_{\mathbb{R}^{n}}, M_{L_{2}}=\max _{t, s \in[0, a]}\left|m_{L_{2}}\right|_{\mathbb{R}^{p}}
$$

and $m_{L_{i}}$ represents the set of continuous selections for the multivalued operator $t \mapsto \lambda_{i 2} \int_{0}^{a} L_{i}\left(t, s, x_{1}(s), x_{2}(s)\right) d s$ for $x_{1} \in C\left(I, \mathbb{R}^{n}\right)$ and $x_{2} \in$ $C\left(I, \mathbb{R}^{p}\right)$.

v) the matrix $I-M$ has the absolute value property, where

$$
M=\left(\frac{\left|\lambda_{i 1}\right| a_{i j}}{\tau}\right)_{i, j=1,2}, \tau>0 .
$$

Then, there exists $\left(x_{1}^{0}, x_{2}^{0}\right) \in C\left(I, \mathbb{R}^{n}\right) \times C\left(I, \mathbb{R}^{p}\right)$ such that our inclusions system has at least one solution $x^{*}:=\left(x_{1}^{*}, x_{2}^{*}\right) \in \bar{B}\left(x_{1}^{0}, R_{1}\right) \times \bar{B}\left(x_{2}^{0}, R_{2}\right) \subset$ $C\left(I, \mathbb{R}^{n}\right) \times C\left(I, \mathbb{R}^{p}\right)$.

Proof. For the sake of simplicity let us denote $X_{1}:=\mathbb{R}^{n}, X_{2}:=\mathbb{R}^{p}$ and $X:=C\left(I, X_{1}\right) \times C\left(I, X_{2}\right)$. Let $F_{1}, G_{1}: X \rightarrow \mathcal{P}\left(C\left(I, X_{1}\right)\right)$ be two multivalued operators given by:

$$
\begin{aligned}
& F_{1}\left(x_{1}, x_{2}\right)=\left\{u \in X: u(t) \in \lambda_{11} \int_{0}^{t} K_{1}\left(t, s, x_{1}(s), x_{2}(s)\right) d s \text { a.e. on } I\right\}, \\
& G_{1}\left(x_{1}, x_{2}\right)=\left\{v \in X: v(t) \in \lambda_{12} \int_{0}^{a} L_{1}\left(t, s, x_{1}(s), x_{2}(s)\right) d s \text { a.e. on } I\right\} .
\end{aligned}
$$

Let $F_{2}, G_{2}: X \rightarrow \mathcal{P}\left(C\left(I, X_{2}\right)\right)$ be two multivalued operators given by:

$$
\begin{aligned}
F_{2}\left(x_{1}, x_{2}\right) & =\left\{u \in X: u(t) \in \lambda_{21} \int_{0}^{t} K_{2}\left(t, s, x_{1}(s), x_{2}(s)\right) d s \text { a.e. on } I\right\}, \\
G_{2}\left(x_{1}, x_{2}\right) & =\left\{v \in X: v(t) \in \lambda_{22} \int_{0}^{a} L_{2}\left(t, s, x_{1}(s), x_{2}(s)\right) d s \text { a.e. on } I\right\} . \\
\text { Then, } F & =\left(\begin{array}{c}
F_{1} \\
F_{2}
\end{array}\right), G=\left(\begin{array}{c}
G_{1} \\
G_{2}
\end{array}\right): X \rightarrow \mathcal{P}(X) .
\end{aligned}
$$


For $x=\left(x_{1}, x_{2}\right) \longrightarrow F(x)=\left(F_{1}(x), F_{2}(x)\right), G(x)=\left(G_{1}(x), G_{2}(x)\right)$. Obviously, $x^{*}=\left(x_{1}^{*}, x_{2}^{*}\right)$ is a solution for our inclusions system if and only if $x^{*}$ is a fixed point for $F(x)+G(x)$. We need to show that the multivalued operators $F$ and $G$ satisfies the assumptions of Theorem 2.1. By Ascoli-Arzelà theorem, we have that $F: X \rightarrow P_{c p, c v}(X)$.

Let $x:=\left(x_{1}, x_{2}\right), y:=\left(y_{1}, y_{2}\right) \in X$ and let $u=\left(u_{1}, u_{2}\right) \in F(x)$. That is $u_{1} \in F_{1}(x), u_{2} \in F_{2}(x)$. Then $u_{1}, u_{2} \in X$ and

$$
\begin{aligned}
& u_{1}(t) \in \lambda_{11} \int_{0}^{t} K_{1}\left(t, s, x_{1}(s), x_{2}(s)\right) d s \text { a.e. on } I, \text { respectively } \\
& u_{2}(t) \in \lambda_{21} \int_{0}^{t} K_{2}\left(t, s, x_{1}(s), x_{2}(s)\right) d s \text { a.e. on } I \text {. }
\end{aligned}
$$

It follows that there exists a mapping $k_{x}^{1} \in S_{K_{1}\left(\cdot, \cdot, x_{1}(\cdot), x_{2}(\cdot)\right)}^{1}$, respectively $k_{x}^{2} \in$ $S_{K_{2}\left(\cdot, \cdot, x_{1}(\cdot), x_{2}(\cdot)\right)}^{1}$ such that

$$
\begin{aligned}
& u_{1}(t) \in \lambda_{11} \int_{0}^{t} k_{x}^{1}(t, s) d s \text { a.e. on } I \text {, respectively } \\
& u_{2}(t) \in \lambda_{21} \int_{0}^{t} k_{x}^{2}(t, s) d s \text { a.e. on } I .
\end{aligned}
$$

For $i \in\{1,2\}$, from the relation

$$
\begin{aligned}
H_{X_{i}}\left(K_{i}\left(t, s, x_{1}(t), x_{2}(t)\right),\right. & \left.K_{i}\left(t, s, y_{1}(t), y_{2}(t)\right)\right) \\
& \leq a_{i 1}\left|x_{1}(t)-y_{1}(t)\right|_{X_{1}}+a_{i 2}\left|x_{2}(t)-y_{2}(t)\right|_{X_{2}}
\end{aligned}
$$

we get that there exists $w_{1} \in K_{1}\left(t, s, y_{1}(t), y_{2}(t)\right)$, respectively $w_{2} \in K_{2}\left(t, s, y_{1}(t), y_{2}(t)\right)$ such that

$\left|k_{x}^{1}(t, s)-w_{1}\right|_{X_{1}} \leq a_{11}\left|x_{1}(t)-y_{1}(t)\right|_{X_{1}}+a_{12}\left|x_{2}(t)-y_{2}(t)\right|_{X_{2}}$, respectively $\left|k_{x}^{2}(t, s)-w_{2}\right|_{X_{2}} \leq a_{21}\left|x_{1}(t)-y_{1}(t)\right|_{X_{1}}+a_{22}\left|x_{2}(t)-y_{2}(t)\right|_{X_{2}}$.

Thus, the multivalued operator $T=\left(T_{1}, T_{2}\right)$, defined by

$$
\begin{aligned}
& T_{1}(t, s)=K_{1}\left(t, s, y_{1}(t), y_{2}(t)\right) \cap K_{T_{1}}(t, s), \\
& T_{2}(t, s)=K_{2}\left(t, s, y_{1}(t), y_{2}(t)\right) \cap K_{T_{2}}(t, s)
\end{aligned}
$$

has nonempty values and is measurable, where

$$
K_{T_{i}}(t, s)=\left\{w_{i}:\left|k_{x}^{i}(t, s)-w_{i}\right| \leq a_{i 1}\left|x_{1}(t)-y_{1}(t)\right|_{X_{1}}+a_{i 2}\left|x_{2}(t)-y_{2}(t)\right|_{X_{2}}\right\},
$$$$
\text { for } i \in\{1,2\} \text {. }
$$ 
Let $k_{y}^{1}$ be a measurable selection for $T_{1}$, respectively $k_{y}^{2}$ be a measurable selection for $T_{2}$ (which exists by Kuratowski-Ryll-Nardzewski's selection theorem, see [7]), then $k_{y}^{1}(t, s) \in K_{1}\left(t, s, y_{1}(t), y_{2}(t)\right)$, respectively $k_{y}^{2}(t, s) \in$ $K_{2}\left(t, s, y_{1}(t), y_{2}(t)\right)$ and

$$
\begin{aligned}
& \left|k_{x}^{1}(t, s)-k_{y}^{1}(t, s)\right|_{X_{1}} \leq a_{11}\left|x_{1}(t)-y_{1}(t)\right|_{X_{1}}+a_{12}\left|x_{2}(t)-y_{2}(t)\right|_{X_{2}}, \\
& \left|k_{x}^{2}(t, s)-k_{y}^{2}(t, s)\right|_{X_{2}} \leq a_{21}\left|x_{1}(t)-y_{1}(t)\right|_{X_{1}}+a_{22}\left|x_{2}(t)-y_{2}(t)\right|_{X_{2}} .
\end{aligned}
$$

We define $v_{1}(t)=\lambda_{11} \int_{0}^{t} k_{y}^{1}(t, s) d s$, respectively $v_{2}(t)=\lambda_{21} \int_{0}^{t} k_{y}^{2}(t, s) d s$. It follows that $v_{1}(t) \in F_{1}(y)$, respectively $v_{2}(t) \in F_{2}(y)$ and for $i \in\{1,2\}$, we have

$$
\begin{aligned}
\mid u_{i}(t) & -\left.v_{i}(t)\right|_{X_{i}} \\
& \leq\left|\lambda_{i 1}\right| \int_{0}^{t}\left|k_{x}^{i}(t, s)-k_{y}^{i}(t, s)\right|_{X_{i}} d s \\
& \leq\left|\lambda_{i 1}\right|\left(a_{i 1} \int_{0}^{t}\left|x_{1}(s)-y_{1}(s)\right|_{X_{1}} d s+a_{i 2} \int_{0}^{t}\left|x_{2}(s)-y_{2}(s)\right|_{X_{2}} d s\right) \\
& =\left|\lambda_{i 1}\right|\left(a_{i 1}|| x_{1}-y_{1}||_{B_{1}} \int_{0}^{t} e^{\tau s} d s+a_{i 2}|| x_{2}-y_{2}||_{B_{2}} \int_{0}^{t} e^{\tau s} d s\right) \\
& \leq \frac{\left|\lambda_{i 1}\right|}{\tau} e^{\tau t}\left(a_{i 1}\left\|x_{1}-y_{1}||_{B_{1}}+a_{i 2}\right\| x_{2}-y_{2} \|_{B_{2}}\right),
\end{aligned}
$$

where $\|u\|_{B}:=\left(\begin{array}{l}\left\|u_{1}\right\|_{B_{1}} \\ \left\|u_{2}\right\|_{B_{2}}\end{array}\right)=\left(\begin{array}{c}\sup _{t \in[0, a]} e^{-\tau t}\left|u_{1}(t)\right|_{X_{1}} \\ \sup _{t \in[0, a]} e^{-\tau t}\left|u_{2}(t)\right|_{X_{2}}\end{array}\right), \tau>0$ denotes the

Bielecki-type norm on the generalized Banach space $X$. Thus, for $i \in\{1,2\}$, we obtain that

$$
\left\|u_{i}-v_{i}\right\|_{B_{i}} \leq \frac{\left|\lambda_{i 1}\right|}{\tau}\left(a_{i 1}\left\|x_{1}-y_{1}\right\|_{B_{1}}+a_{i 2}\left\|x_{2}-y_{2}\right\|_{B_{2}}\right)
$$

and similarly, interchanging the roles between $x$ and $y$, for $i \in\{1,2\}$, we get that

$$
H_{B_{i}}\left(F_{i}(x), F_{i}(y)\right) \leq \frac{\left|\lambda_{i 1}\right|}{\tau}\left(a_{i 1}\left\|x_{1}-y_{1}\right\|_{B_{1}}+a_{i 2}\left\|x_{2}-y_{2}\right\|_{B_{2}}\right),
$$

for each $x, y \in X$.

These inequalities can be written in the matrix form

$$
H_{B}(F(x), F(y)) \leq M\|x-y\|_{B},
$$


where

$$
M=\left(\frac{\left|\lambda_{i 1}\right| a_{i j}}{\tau}\right)_{i, j=1,2} .
$$

Taking $\tau$ large enough it follows that the matrix $M$ is convergent to zero and thus, $F$ is a multivalued $M$-contraction. By Theorem 1.6, we have that there exists a fixed point $x^{0}=\left(x_{1}^{0}, x_{2}^{0}\right) \in X$ for $F$, i.e. $x^{0} \in F\left(x^{0}\right)$. That is $x_{1}^{0} \in F_{1}\left(x^{0}\right)$ and $x_{2}^{0} \in F_{2}\left(x^{0}\right)$.

Let us consider $Y=\bar{B}\left(x_{1}^{0}, R_{1}\right) \times \bar{B}\left(x_{2}^{0}, R_{2}\right) \subset C\left(I, X_{1}\right) \times C\left(I, X_{2}\right)$. We show that $F(Y) \subset \bar{B}\left(x_{1}^{0}, \frac{R_{1}}{2}\right) \times \bar{B}\left(x_{2}^{0}, \frac{R_{2}}{2}\right)$. Let $x \in Y$, then $\left(x_{1}, x_{2}\right) \in$ $\bar{B}\left(x_{1}^{0}, R_{1}\right) \times \bar{B}\left(x_{2}^{0}, R_{2}\right)$. Thus,

$$
\left\|x_{1}-x_{1}^{0}\right\|_{C_{1}} \leq R_{1} \text { and }\left\|x_{2}-x_{2}^{0}\right\|_{C_{2}} \leq R_{2}
$$

Let $u=\left(u_{1}, u_{2}\right) \in F(x)$ be arbitrarily chosen. That is $u_{1} \in F_{1}(x), u_{2} \in$ $F_{2}(x)$. Then $u_{1}(t) \in \lambda_{11} \int_{0}^{t} K_{1}\left(t, s, x_{1}(s), x_{2}(s)\right) d s$, respectively $u_{2}(t) \in$ $\lambda_{21} \int_{0}^{t} K_{2}\left(t, s, x_{1}(s), x_{2}(s)\right) d s \quad$ a.e. $\quad$ on $\quad I$. It follows that $u_{i}(t)=\lambda_{i 1} \int_{0}^{t} k_{x}^{i}(t, s) d s$, where $k_{x}^{i}(t, s) \in K_{i}\left(t, s, x_{1}(s), x_{2}(s)\right)$ a.e. on $I^{2}$, for $i \in\{1,2\}$. We have

$$
\begin{aligned}
\mid u_{i}(t) & -\left.x_{i}^{0}(t)\right|_{X_{i}} \\
& \leq\left|\lambda_{i 1}\right| \int_{0}^{t}\left|k_{x}^{i}(t, s)-k_{x^{0}}^{i}(t, s)\right|_{X_{i}} d s \\
& \leq\left|\lambda_{i 1}\right|\left(a_{i 1} \int_{0}^{t}\left|x_{1}(s)-x_{1}^{0}(s)\right|_{X_{1}} d s+a_{i 2} \int_{0}^{t}\left|x_{2}(s)-x_{2}^{0}(s)\right|_{X_{2}} d s\right) \\
& \leq\left|\lambda_{i 1}\right| \int_{0}^{t}\left(a_{i 1}|| x_{1}-x_{1}^{0}||_{C_{1}}+a_{i 2}|| x_{2}-x_{2}^{0}||_{C_{2}}\right) \\
& \leq\left|\lambda_{i 1}\right| a\left(a_{i 1} R_{1}+a_{i 2} R_{2}\right), \text { for } i \in\{1,2\} .
\end{aligned}
$$

Taking $\max _{t \in[0, a]}\left|u_{i}(t)\right|_{X_{i}}$, we get that

$$
\left\|u_{i}-x_{i}^{0}\right\|_{C_{i}} \leq\left|\lambda_{i 1}\right| a\left(a_{i 1} R_{1}+a_{i 2} R_{2}\right) \leq \frac{R_{i}}{2}, \text { for } i \in\{1,2\}
$$

The multivalued operator $G$ is l.s.c. and compact.

We show that $G(Y)$ is relatively compact.

Let $x:=\left(x_{1}, x_{2}\right) \in Y$ and we prove that $G(Y) \subset \bar{B}\left(0, \frac{R_{1}}{2}\right) \times \bar{B}\left(0, \frac{R_{2}}{2}\right)$. Let $v=\left(v_{1}, v_{2}\right) \in G(x)$ be arbitrarily chosen. That is $v_{1} \in G_{1}(x)$, respectively $v_{2} \in G_{2}(x)$. Then $v_{1}(t) \in \lambda_{12} \int_{0}^{a} L_{1}\left(t, s, x_{1}(s), x_{2}(s)\right) d s$, respectively $v_{2}(t) \in \lambda_{22} \int_{0}^{a} L_{2}\left(t, s, x_{1}(s), x_{2}(s)\right) d s$ a.e. on $I$. It follows that 
$v_{i}(t)=\lambda_{i 2} \int_{0}^{a} l_{x}^{i}(t, s) d s$, where $l_{x}^{i}(t, s) \in L_{i}\left(t, s, x_{1}(s), x_{2}(s)\right)$ a.e. on $I^{2}$, for $i \in\{1,2\}$. Clearly,

$$
\left|v_{i}(t)\right|_{X_{i}} \leq\left|\lambda_{i 2}\right| \int_{0}^{a}\left|l_{x}^{i}(t, s)\right|_{X_{i}} d s \leq\left|\lambda_{i 2}\right| a M_{L_{i}} \leq \frac{R_{i}}{2} \text {, for } i \in\{1,2\} .
$$

Thus, $v_{1} \in \bar{B}\left(0, \frac{R_{1}}{2}\right), v_{2} \in \bar{B}\left(0, \frac{R_{2}}{2}\right)$. Then, the multivalued operator $U=$ $F+G$ has the property $U: Y \rightarrow P_{c p, c v}(Y)$, i.e. $F(x)+G(x) \subset Y$, for each $x \in Y$. Hence, the conclusion follows by Theorem 2.1 .

Remark 2.3. The above Theorem can be improved by supposing instead of the existence of a real positive number square matrix $A$, another square matrix $A=\left(a_{i j}\right)_{i, j=1,2}$, where $a_{i j} \in L^{p}\left([0, a], \mathbb{R}_{+}\right), i, j \in\{1,2\}$ and using the Hölder's inequality, we can obtain too, another similar result.

Acknowledgement. The author wishes to thank for the financial support provided from programs co-financed by The Sectoral Operational Programme Human Resources Development, Contract POSDRU/88/1.5/S/ 60185 - "Innovative Doctoral Studies in a Knowledge Based Society".

\section{References}

[1] G. Allaire and S.M. Kaber, Numerical Linear Algebra, Texts in Applied Mathematics, 55(2008), Springer, New York.

[2] A. Bucur, L. Guran, A. Petruşel, Fixed points for multivalued operators on a set endowed with vector-valued metrics and applications, Fixed Point Theory, 10(2009), No. 1, 19-34.

[3] T.A. Burton, A fixed-point theorem of Krasnoselskii, Appl. Math. Letters, 11(1998), 85-88.

[4] S. Carl, S. Heikkila, Fixed Point Theory in Ordered Sets and Applications, Springer, New York, 2011.

[5] L. Collatz, Some applications of functional analysis to analysis, particularly to nonlinear integral equations, Nonlinear Functional Anal. and Appl. (Proc. Advanced Sem., Math. Res. Center, Univ. of Wisconsin, Madison, Wis, 1970), Academic Press, New York, 1-43.

[6] H. Covitz, S.B. Nadler Jr., Multivalued contraction mappings in generalized metric spaces, Israel J. Math., 8(1970), 5-11.

[7] K. Deimling, Multivalued differential equations, W. de Gruyter, 1992. 
[8] A.-D. Filip, A. Petrusel, Fixed point theorems on spaces endowed with vector-valued metrics, FPTA, 2010(2010), Article ID 281381, 15 pp.

[9] A. Granas, J. Dugundji, Fixed Point Theory, Springer, New York, 2003.

[10] D. Guo, V. Lakshmikantham, X. Liu, Nonlinear Integral Equations in Abstract Spaces, Kluwer Academic Publisher, Dordrecht, 1996.

[11] W.A.J. Luxemburg, A.C. Zaanen, Riesz Spaces, North-Holland Publishing Company, Amsterdam, 1(1971).

[12] I. Muntean, Capitole Speciale de Analiză Funcţională, Cluj-Napoca, 1990 (in Romanian).

[13] I. Muntean, În legătură cu o teoremă de punct fix în spaţii local convexe, Rev. Roumaine Math. Pures Appl., 19(1974), 1105-1109 (in Russian).

[14] S.B. Nadler Jr., Multi-valued contraction mappings, Pacific J. Math., 30(1969), 475-487.

[15] D. O'Regan, N. Shahzad, R.P. Agarwal, Fixed point theory for generalized contractive maps on spaces with vector-valued metrics, Fixed Point Theory and Applications, Nova Sci. Publ., New York, 6(2007), 143-149.

[16] D. O'Regan, Fixed-point theory for the sum of two operators, Appl. Math. Let., 9(1996), 1-8.

[17] A.I. Perov, On the Cauchy problem for a system of ordinary differential equations, Priblizhen. Metody Reshen. Differ. Uravn., 2(1964), 115-134 (in Russian).

[18] A.I. Perov, A.V. Kibenko, On a certain general method for investigation of boundary value problems, Izv. Akad. Nauk SSSR Ser. Mat., 30(1966), 249-264 (in Russian).

[19] I.-R. Petre, A. Petruşel, Krasnoselskii's theorem in generalized Banach spaces and applications, Electron. J. Qual. Theory Differ. Equ., 85(2012), $1-20$.

[20] A. Petruşel, A generalization of Krasnoselskii's fixed point theorem, Proc. Sci. Comm. Metting of "Aurel Vlaicu" Univ. Arad, Vol. 14A(1996), 109112.

[21] A. Petruşel, Integral Inclusions. Fixed point approaches, Annales Soc. Math. Pol., Series I: Commentiones Mathematicae XL, 2000, 147-158. 
[22] A. Petrussel, Multivalued operators and fixed points, Pure Math. Appl., 11(2000), No. 2, 361-368.

[23] A. Petruşel, Multivalued weakly Picard operators and applications, Sci. Math. Jap., 59(2004), No. 1, 169-202.

[24] A. Petrussel, I.A. Rus, The theory of a metric fixed point theorem for multivalued operators, Fixed Point Theory and its Applications, Yokohama Publ., 2010, 167-176.

[25] R. Precup, A. Viorel, Existence results for systems of nonlinear evolution equations, Intern. J. Pure Appl. Math., Vol. 47(2008), No. 2, 199-206.

[26] R. Precup, A. Viorel, Existence results for systems of nonlinear evolution inclusions, Fixed Point Theory, 11(2010), No. 2, 337-346.

[27] R. Precup, Methods in Nonlinear Integral Equations, Kluwer, Dordrecht, 2002.

[28] R. Precup, The role of matrices that are convergent to zero in the study of semilinear operator systems, Mathematical and Computer Modelling, 49(2009), No. 3-4, 703-708.

[29] L. Rybinski, An application of the continuous selection theorem to the study of the fixed points of multivalued mappings, J. Math. Anal. Appl., 153(1990), 391-396.

[30] I.A. Rus, A. Petruęl, A. Sîntămărian, Data dependence of the fixed point set of multivalued weakly Picard operators, Nonlinear Anal., 52(2003), 1947-1959.

[31] I.A. Rus, A. Petruşel, G. Petruşel, Fixed Point Theory, Cluj University Press, Cluj-Napoca, 2008.

[32] I.A. Rus, Generalized Contractions and Applications, Cluj University Press, Cluj-Napoca, 2001.

[33] I.A. Rus, Principles and Applications of the Fixed Point Theory, Dacia, Cluj-Napoca, 1979 (in Romanian).

[34] I.A. Rus, Technique of the fixed point structures for multivalued mappings, Math. Japonica, 38(1993), 289-296.

[35] R.S. Varga, Matrix Iterative Analysis, Vol. 27 of Springer Series in Computational Mathematics, Springer-Verlag, Berlin, 2000. 
[36] A. Viorel, Contributions to the study of nonlinear evolution equations, Ph.D. Thesis, Babeş-Bolyai University Cluj-Napoca, 2011.

[37] P.P. Zabrejko, K-metric and K-normed linear spaces: survey, Collect. Math., 48(1997), No. 4-6, 825-859.

[38] A.C. Zaanen, Riesz Spaces, North-Holland Publishing Company, Amsterdam, 2(1983).

[39] E. Zeidler, Nonlinear Functional Analysis, Vol. I, Fixed Point Theorems, Springer-Verlag, Berlin, 1993.

[40] M. Zuluaga, On a fixed point theorem and application to a two-point boundary value problem, Comment. Math. Univ. Carolinae, 27(1986), 731735 .

Ioan-Radu PETRE

Department of Applied Mathematics,

University of Babeş-Bolyai,

Kogălniceanu No. 1, 400084, Cluj-Napoca, România.

Email: ioan.petre@ubbcluj.ro 